\title{
Análise Cristalográfica da Etoxi-Chalcona Fluorada $\mathrm{C}_{17} \mathrm{H}_{15} \mathrm{O}_{2} \mathrm{~F}$
}

\author{
Cássio C. Almeida, Ademir J. Camargo, Gilberto B. Aquino \& \\ Hamilton B. Napolitano.
}

Chalconas é uma importante classe de flavonoides e apresenta interessantes atividades biológicas e, em particular, as chalconas fluoradas por possuir ação anticarcinogênica. A substituição de uma ligação $\mathrm{C}-\mathrm{H}$ ou $\mathrm{C}-\mathrm{OH}$ por $\mathrm{C}-\mathrm{F}$ é frequente em compostos biologicamente ativos, pois estas substituições resultam em alterações nas propriedades físico-químicas da molécula sem, no entanto, produzir maior mudança estérica. Este projeto elucidou a molécula $\mathrm{C}_{17} \mathrm{H}_{15} \mathrm{O}_{2} \mathrm{~F}$ a partir da mistura de 4-fluor-acetofenona com 4-fluorbenzaldeído e $\mathrm{NaOH}$. Foi utilizada na análise da molécula a cristalografia, como método científico a fim de se conhecer a estrutura molecular e cristalina, através da difração de raios-X, e para entender o problema conformacional utilizou-se a Density Functional Theory (DFT). Desta maneira este estudo apresenta uma nova chalcona fluorada com Z'>1 (conformações $\alpha$ e $\beta$ ). A estrutura da chalcona $\mathrm{C}_{17} \mathrm{H}_{15} \mathrm{O}_{2} \mathrm{~F}$ é similar com estruturas de chalconas que apresentam atividade biológica anticarcinogênica.

Palavras Chave: chalconas; difração de raios-X; DFT.

Chalcones are an important class of flavonoids and possess interesting biological activities, in particular fluorinated chalcones to possess anticarcinogenic action. The replacement of a $\mathrm{C}-\mathrm{H}$ bond or $\mathrm{C}-\mathrm{C}-\mathrm{OH}$ of $\mathrm{F}$ is common in biologically active compounds as such substitutions result in a change in the physicochemical properties of the molecule without, however, produce more steric changes. This design attempts elucidated the molecular $\mathrm{C}_{17} \mathrm{H}_{15} \mathrm{O}_{2} \mathrm{~F}$ from the mixture of 4-fluoro-acetophenone with 4-fluorbenzaldehyde and $\mathrm{NaOH}$. It was used in the analysis of the molecule crystallography as scientific method in order to know the molecular and crystal structure by X-ray diffraction, and to understand the problem we used the conformation Density Functional Theory (DFT). Thus this study presents a new fluorinated chalcone with $Z^{\prime}>1$ ( $\alpha$ and $\beta$ conformations). The structure is similar to chalcone $\mathrm{C}_{17} \mathrm{H}_{15} \mathrm{O}_{2} \mathrm{~F}$ chalcones structures that exhibit biological activity anticarcinogenic.

Keywords: chalcones, $X$-ray diffraction; $D F T$. 


\section{Introdução}

$\mathrm{O}$ grande desafio de pesquisadores e das grandes indústrias farmacêuticas vem sendo a busca de medicamentos que possam curar as mais variadas doenças e que possuam o mínimo de efeitos colaterais. Atualmente, a descoberta de novas moléculas bioativas segue um planejamento mais racional, no qual, a interdisciplinaridade permite construir protótipos de fármacos de maneira mais direta, sem que haja a experimentação aleatória. Estudos relacionando síntese, identificação e modificação estrutural de novas moléculas de interesse químico e medicinal, avaliam suas propriedades, estabelecendo uma relação entre estrutura química e atividade biológica para obtenção de compostos de potente aplicabilidade terapêutica.

Dentro deste contexto, as chalconas são compostos que se destacam visto sua diversidade estrutural e praticidade sintética, sendo definidas como cetonas $\alpha$ e $\beta$, insaturadas, em que a carbonila e o fragmento olefínico estão ligados a grupamentos aromáticos, comumente designados como anel A, proveniente da cetona, e anel B, proveniente do aldeído, conforme Figura 1. Estudos sobre os avanços terapêuticos das chalconas continuam sendo desenvolvidos na busca de moléculas potencialmente ativas.

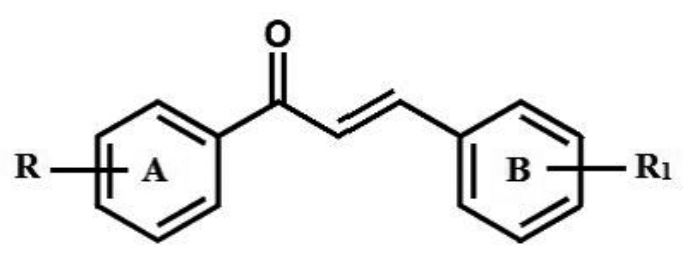

Figura 1. Fórmula estrutural básica das chalconas.

O conhecimento do arranjo dos átomos em uma molécula (estrutura molecular) e da posição relativa de todas as moléculas em um cristal (estrutura cristalina), para substâncias obtidas tanto sinteticamente como isoladas de fontes naturais, é extremamente útil para o entendimento das propriedades químicas, físico-químicas e biológicas dos compostos para os mais variados ramos da ciência .

Os derivados de chalcona têm atraído a atenção cada vez maior devido a um amplo espectro de aplicações farmacológicas. Chalconas é uma importante classe de flavonoides e apresenta interessantes atividades biológicas, incluindo anticarcinogênica, antioxidante, anti-inflamatória, citotóxica, antimicrobiana, analgésica antipirética, anti-anginosos, anti-hepatotóxica, antimaláricos e antialérgico. Os flavonoides compõem uma ampla classe de substâncias de origem natural, cuja síntese não ocorre na espécie humana. Entretanto, tais compostos possuem uma série de propriedades farmacológicas que os fazem atuar sobre sistemas biológicos. Consequentemente, muitas dessas propriedades atuam de forma benéfica para a saúde humana. Atualmente, já foram identificadas mais de quatro mil substâncias pertencentes ao grupo dos flavonoides. A causa para a existência de uma grande diversidade estrutural dos flavonoides é explicada pelas modificações que tais compostos podem sofrer, como: hidroxilação, metilação, acilação, glicosilação, entre outras. Devido à sua versatilidade sintética e eficazes atividades biológicas, estes compostos têm provocado grande interesse para possíveis usos terapêuticos.

A estrutura molecular (E)-3-(4-etoxifenil)-1-(4-fluorfenil) prop-2-en-1-ona, $\left(\mathrm{C}_{17} \mathrm{H}_{15} \mathrm{O}_{2} \mathrm{~F}\right)$, ilustrada na (Figura 2), foi sintetizada por reação de condensação de Claisen-Schmidt e cristalizada pelo método de evaporação lenta; teve seus dados obtidos pelo difratômetro Kappa Charge Coupled Device (CCD), com radiação monocromática Moka, sendo caracterizado por difração de raios-X de monocristal e cálculo Density Functional Theory (DFT), ajudando a compreender a determinação da estrutura cristalina, explorando a configuração e conformação supramolecular.

Esta molécula cristaliza-se no grupo espacial centrossimétrico com duas conformações independentes na unidade assimétrica, mostrando algumas características relacionadas com moléculas de sistemas estruturais de Z'>1; como: baixa simetria, multiplicidade conformacional e geométrica, interações fracas, como também as interações estruturais aromáticas.

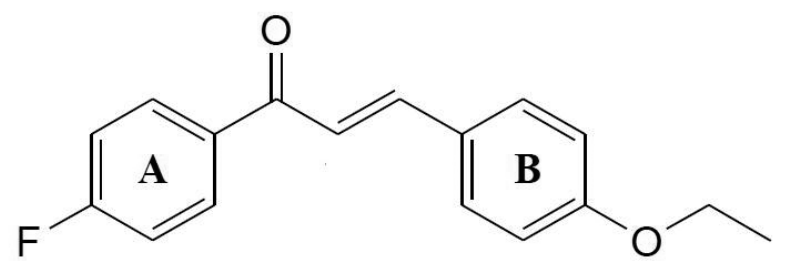

Figura 2. Fórmula estrutural da chalcona $\mathrm{C}_{17} \mathrm{H}_{15} \mathrm{O}_{2} \mathrm{~F}$ 


\section{Estudo da Chalcona \\ $\mathrm{C}_{17} \mathrm{H}_{15} \mathrm{O}_{2} \mathrm{~F}$}

\section{PROCEDIMENTOS EXPERIMENTAIS}

Achalcona, $\mathrm{C}_{17} \mathrm{H}_{15} \mathrm{O}_{2} \mathrm{~F}$, foi preparada pela condensação aldólica de Claisen-Schmidt pela reação da mistura de 4-Fluor-acetofenona (0,3 mg, $2 \mathrm{mmol})$ com 4, etoxibenzaldeído (0,39 mg, $2 \mathrm{mmol}$ ) e $\mathrm{NaOH}$ (50\% p / v) em metanol a temperatura $293 \mathrm{~K}$ durante 24 horas (Figura 3 ). O sólido amarelo claro (m.p. 404.25-405.65 K) obtido foi filtrado, lavado com água e seco.

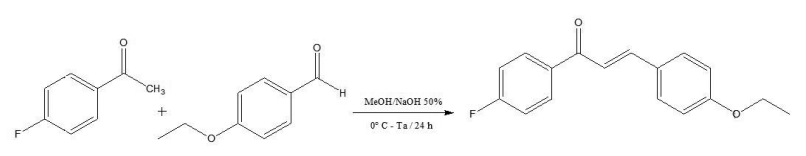

Figura 3. Representação do esquema de síntese da chalcona $\mathrm{C}_{17} \mathrm{H}_{15} \mathrm{O}_{2} \mathrm{~F}$ via catálise básica

O processo de formação de cristais foi realizado dentro do Laboratório de Enzimologia da Unidade Universitária de Ciências Exatas e Tecnológicas (UNUCET) da UEG de Anápolis, cujo método utilizado foi o de evaporação lenta. Os monocristais obtidos, com qualidade adequada para difração de raios-X, foram cultivados em metanol. Os dados de difração de raios- $X$ foram coletados a temperatura ambiente, utilizando um Difratômetro KAPPA-CCD com MoK $\alpha$ radiação $(\lambda=0,71073 \AA)$.

A estrutura foi resolvida por Métodos Diretos e anisotropicamente refinado com matriz completa de mínimos quadrados, minimizando as diferenças entre fatores de estrutura, observados e calculados em $\mathrm{F}^{2}$, usando SHELXL97 do pacote de programas WingX. Os átomos de hidrogênio foram colocados em posições calculadas e refinados, eliminando restrições. Os dados cristalográficos foram depositados no CCDC, sob os números 5689 CCDC. Cópias dos dados podem ser obtidas, gratuitamente, por meio do site www.ccdc.cam.ac.uk. A estrutura eletrônica da unidade assimétrica foi investigada pela DFT. Todos os cálculos foram realizados, utilizando o Programa Gaussian
03. Os parâmetros otimizados da chalcona $\mathrm{C}_{17} \mathrm{H}_{15} \mathrm{O}_{2} \mathrm{~F}$, (comprimentos de ligação, ângulos de ligação e ângulos torsão) foram obtidos através do conjunto de base 6-311+ $+\mathrm{g}(\mathrm{d}, \mathrm{p})$ e três parâmetros híbridos(B3LYP), denotando a combinação do funcional de troca de Becke com o funcional de correlação de Lee, Yang e Parr. A varredura fixa da curva do potencial de energia, em torno do grupo C6-fluorfenil, foi realizada, variando o ângulo de torção C6 $-\mathrm{C} 7-\mathrm{C} 8=\mathrm{C} 9$ com uma escala de $10^{\circ}$, partindo de $0^{\circ}$ a $360^{\circ}$.

$\mathrm{Na}$ varredura fixa em torno do grupo C6-fluor, as contribuições da energia do Zero Point Vibrational Energy (ZPVE) são insignificantes. Posição de hidrogênio, das duas conformações, foi modificada para uma melhor descrição de uma barreira energética. O mínimo local na hipersuperfície da energia potencial foi caracterizado pelos cálculos das frequências de vibração. As cargas parciais atômicas calculadas neste trabalho foram derivadas do Molecular Electrostatic Potencial (MEP), utilizando o formalismo (CHELPG) desenvolvido por Breneman e Wiberg. Neste método, as cargas atômicas são ajustadas para reproduzir o MEP em vários pontos em torno da molécula. Todas as propriedades acima foram calculadas com nível semelhante de cálculo da otimização. As energias de ligação corrigidas para a interação da conformação na unidade assimétrica foram calculadas de acordo com a equação: $\Delta \mathrm{E}=[\mathrm{E}(\mathrm{C} 12)+\mathrm{E}$ (C12) ZPVE] - [E (C1) BSSE + E (C1) ZPVE + E (C2) $\mathrm{BSSE}+\mathrm{E}(\mathrm{C} 2) \mathrm{ZPVE}]$, em que $\triangle \mathrm{E}$ representa a energia de interação, C12 representa a disposição da unidade assimétrica, e C1 e C2 são conformações do composto.

\section{ESTRUTURA CRISTALOGRÁFICA DA CHALCONA $\mathrm{C}_{17} \mathbf{H}_{15} \mathbf{O}_{2} \mathbf{F}$}

A chalcona $\mathrm{C}_{17} \mathrm{H}_{15} \mathrm{O}_{2} \mathrm{~F}$, cristalizada no grupo espacial centrossimétrico $\mathrm{P}_{\overline{1}}$ com duas conformações independentes ( $\alpha$ e $\beta$ ) na unidade assimétrica. Em geral, as características gerais de $Z^{\prime}>1$ são: baixa simetria do cristal e multiplicidade molecular conformacional. A molécula é um chalcona com anéis de 4-fluorfenil 4-etoxifenil ligadas nas extremidades opostas do grupo $-\mathrm{CO}-\mathrm{CH}=\mathrm{CH}-$. A Figura 4 mostra a representação Ortep da unidade assimétrica da estrutura com elipsoides a $50 \%$ de probabilidade, e a Figura 5 mostra a unidade molecular da conformação $\alpha$ (Ortep). 


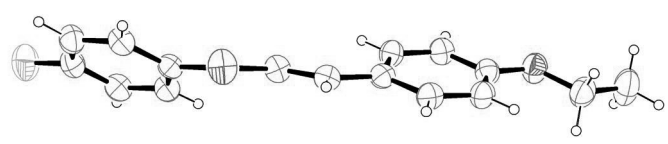

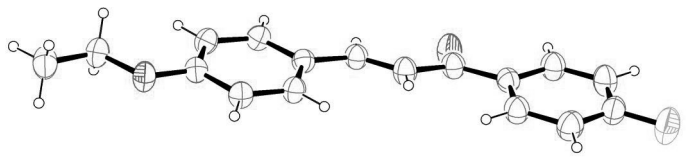

Figura 4. Representação Ortep da unidade assimétrica, com 50\% de probabilidade, ilustrando duas moléculas independentes da chalcona $\mathrm{C}_{17} \mathrm{H}_{15} \mathrm{O}_{2} \mathrm{~F}$.

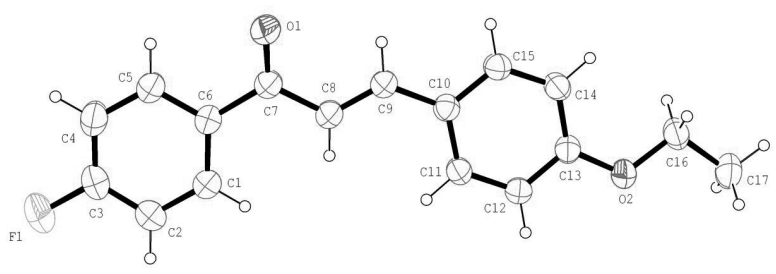

Figura 5. Representação Ortep da conformação $\alpha$ da chalcona $\mathrm{C}_{17} \mathrm{H}_{15} \mathrm{O}_{2} \mathrm{~F}$.

As conformações sobrepostas (Figura 6) mostram diferenças significativas no ângulo diedral C6-C7$\mathrm{C} 8=\mathrm{C} 9$, indicando a orientação do grupo fenil fluorado. A conformação $\alpha, \mathrm{C} 6-\mathrm{C} 7-\mathrm{C} 8=\mathrm{C} 9$, é uma molécula planar, com $-177,16^{\circ}$, e os dois anéis de benzeno formam um ângulo diedral de $6,53^{\circ}$. A rotação livre em torno de $\mathrm{C} 6-\mathrm{C} 7$, em sistemas conjugados, aceitou a hipótese de que a conformação plana maximizou a estrutura. A conformação $\beta, \mathrm{C} 6^{\prime}-\mathrm{C} 7^{\prime}-\mathrm{C} 8^{\prime}=\mathrm{C} 9^{\prime}$, com $-170,91^{\circ}$, e os anéis aromáticos são significativamente distorcidos por $48,90^{\circ}$, em relação um ao outro. Em ambas as moléculas, a ligação $\mathrm{C} 8=\mathrm{C} 9$ tem configuração trans no grupo fenil etoxilado prop-2en-1-ona, sendo essencialmente planar; C14-C13-O2-C16 $\left(1,21^{\circ}\right.$ e $\left.4,5^{\circ}\right)$ para as conformações $\alpha$ e $\beta$, respectivamente; e $\mathrm{C} 13-\mathrm{O} 2-\mathrm{C} 16-\mathrm{C} 17 \quad\left(-176,61^{\circ}\right.$ e $175,45^{\circ}$ ) para as conformações $\alpha$ e $\beta$, respectivamente; com desvios máximos de $0,0256^{\circ}$.

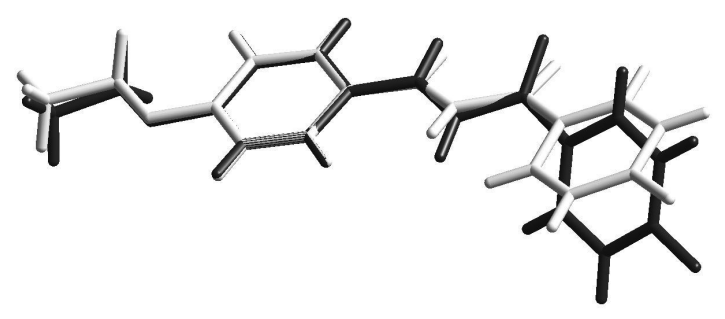

Figura 6. Sobreposição das conformações independentes observadas na unidade assimétrica para a chalcona $\mathrm{C}_{17} \mathrm{H}_{15} \mathrm{O}_{2} \mathrm{~F}$. A conformação $\alpha$ está representada em branco, e a conformação $\beta$ em cinza.

A comparação estrutural, de chalconas similares, feitas através do programa Mogul no Cambridge Structural Database (CSD), para o ângulo diedral C7$\mathrm{C} 8=\mathrm{C} 9-\mathrm{C} 10$ (Figura 7), mostra um valor preferencial, coincidindo com o da conformação $\alpha\left(177,90^{\circ}\right)$.

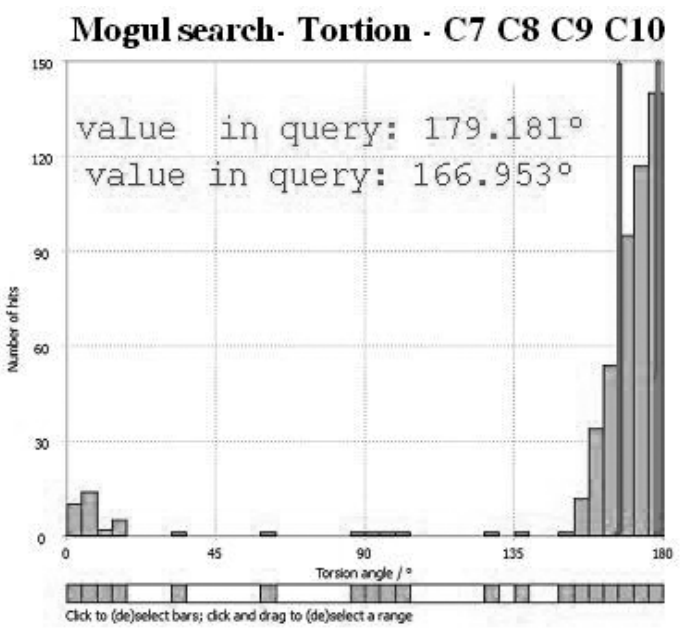

Figura 7. Resultado, em forma de histograma, da busca realizada no CSD pelo programa 
As informações adicionais da determinação da estrutura cristalina são dadas na Tabela 1 .

Tabela 1. Dados cristalográficos e refinamento de estrutura

\begin{tabular}{|l|c|}
\hline Massa Molecular & 270,3 u.a \\
\hline Temperatura & $293 \mathrm{~K}$ \\
\hline Comprimento de Onda & $0,71073 \AA$ \\
\hline Sistema Cristalino & Triclínico \\
\hline Grupo espacial & $\mathrm{P}^{-}$ \\
\hline & $\mathrm{a}=5,85340(2) \AA$ \\
\cline { 2 - 2 } & $\mathrm{b}=15,6909(3) \AA$ \\
\cline { 2 - 2 } & $\mathrm{c}=16,4499(3) \AA$ \\
\cline { 2 - 2 } & $\alpha=66,9110^{\circ}(2)$ \\
\cline { 2 - 2 } & $\beta=87,6500^{\circ}(2)$ \\
\cline { 2 - 2 } & $\gamma=88,9080^{\circ}(2)$. \\
\hline Volume & $1388,64 \mathrm{~A} 3$ \\
\hline Z & 4 \\
\hline$Z^{\prime}$ & 2 \\
\hline Densidade calculada & $1,29 \mathrm{mg} / \mathrm{m} 3$ \\
\hline Coeficiente de absorção & $0,093 \mathrm{~mm}-1$ \\
\hline F(000) & 568 \\
\hline Reflexões coletadas independentes & $29815 / 5667$ \\
\hline Métodos de refinamento & 0,099 \\
\hline Goodness-of-fit on F2 & 1,045 \\
\hline Índice final de R [I>2 $\sigma(\mathrm{I})]$ & Mínos $\mathrm{Quadrados}$ \\
\hline Í́ndices (todos os dados) & \\
\hline
\end{tabular}

$\mathrm{Na}$ estrutura cristalina, $\mathrm{C}_{17} \mathrm{H}_{15} \mathrm{O}_{2} \mathrm{~F}$, as duas moléculas independentes têm configuração supramolecular (Figura 8) formada por interações $\mathrm{CH}$...O, CH...F e $\mathrm{C}-\mathrm{H} . . . \pi$ (Tabela 2).

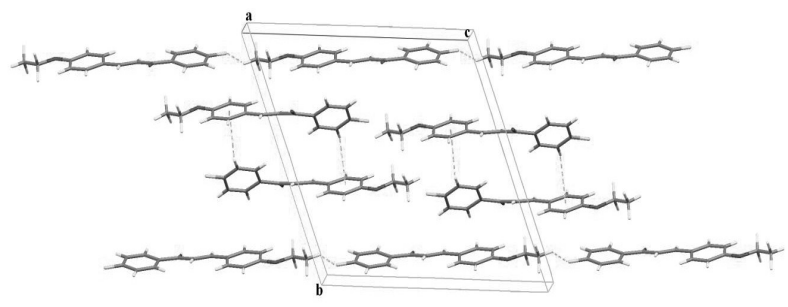

Figura 8. Empacotamento cristalino da chalcona $\mathrm{C}_{17} \mathrm{H}_{15} \mathrm{O}_{2} \mathrm{~F}$.
A conformação $\alpha$ organiza uma cadeia de moléculas ao longo do eixo c por C17-H17A... F1 ${ }^{\text {iiii }}$ [D...a distância $=3,596$ e código de simetria: (iii) $-\mathrm{x}+1, \mathrm{y}-,-\mathrm{z}+1]$. Estas moléculas formam uma rede com $\mathrm{C} 2-\mathrm{H} 2 \ldots \mathrm{O} 2, \mathrm{C} 8-\mathrm{H} 8 \ldots$ O1. A ação recíproca entre estas interações desempenha um papel importante na determinação da conformação desta molécula. Na conformação $\beta$, a distorção de $6,28^{\circ}$ no ângulo de torsão, $\mathrm{C} 6-\mathrm{C} 7-\mathrm{C} 8=\mathrm{C}$, resultado de ligações $\mathrm{CH} . . . \pi$ centrossimétrico que são reproduzidas pela $\mathrm{C} 17^{\prime}-$ H17B...F1', formando uma camada de rede ao longo do eixo c. Observa-se que as estruturas com $Z^{\prime}=2$ têm um empacotamento flexível, mas boa interação resultante na interceptação das camadas de rede da conformação. A Tabela 2 lista as interações de hidrogênio não clássicas principais da estrutura cristalina, $\mathrm{C}_{17} \mathrm{H}_{15} \mathrm{O}_{2} \mathrm{~F}$. A nucleação e a estabilidade molecular são fatores importantes na formação de uma fase sólida estável com Z'>1. O cristal composto pelas conformações $\alpha$ e $\beta$ está em equilíbrio, entre as forças de atração e repulsão, gerando um arranjo estável.

Tabela 2. Interações de hidrogênio não clássicas da chalcona $\mathrm{C}_{17} \mathrm{H}_{15} \mathrm{O}_{2} \mathrm{~F}$.

\begin{tabular}{|c|c|c|c|}
\hline D-H...A & D...A & H...A & D-H...A \\
\hline $\mathrm{C} 8-\mathrm{H} 8 \ldots \mathrm{O} 1 \mathrm{i}$ & $3,766(2)$ & $2,923(1)$ & $151,40(1)$ \\
\hline C16-H16B...O1' iii & $3,731(3)$ & $2,838(2)$ & $153,45(2)$ \\
\hline $\mathrm{C} 2-\mathrm{H} 2 \ldots \mathrm{O} 2 \mathrm{iv}$ & $3,623(2)$ & $2,796(1)$ & $148,75(1)$ \\
\hline C17-H17A...F1iv & $3,805(2)$ & $2,974(1)$ & $145,60(2)$ \\
\hline $\mathrm{C} 1{ }^{\prime}-\mathrm{H} 11^{\prime} . . . \mathrm{O} 1^{\prime} \mathrm{v}$ & $3,764(2)$ & $2,990(1)$ & $141,64(1)$ \\
\hline $\mathrm{C} 8^{\prime}-\mathrm{H} 8^{\prime} . . . \mathrm{O} 1^{\prime} \mathrm{v}$ & $3,692(2)$ & $2,791(1)$ & $163,47(1)$ \\
\hline $\mathrm{C} 11^{\prime}-\mathrm{H} 11^{\prime} \ldots \mathrm{O} 11^{\prime} \mathrm{v}$ & $3,618(2)$ & $2,858(1)$ & $139,76(1)$ \\
\hline C17'-H17E...F1'vi & $3,600(3)$ & $2,979(1)$ & $123,58(1)$ \\
\hline $\mathrm{C} 2^{\prime}-\mathrm{H} 2^{\prime} \ldots \mathrm{Cg} 1$ & $3,491(2)$ & $2,861(2)$ & $126,15^{\circ}(2)$ \\
\hline
\end{tabular}

No estado solúvel, as moléculas são independentes e toda conformação está igualmente provável a ocorrência. As razões para Z' alta são originados no fenômeno de cristalização, em que as moléculas podem adotar diferentes conformações no estado solúvel. Assim, é necessário compreender os parâmetros moleculares energéticos e termodinâmicos. 
As Figuras, 9, 10 e 11 representam as interações de hidrogênio não clássicas da chalcona $\mathrm{C}_{17} \mathrm{H}_{15} \mathrm{O}_{2} \mathrm{~F}$, listadas na Tabela 2.

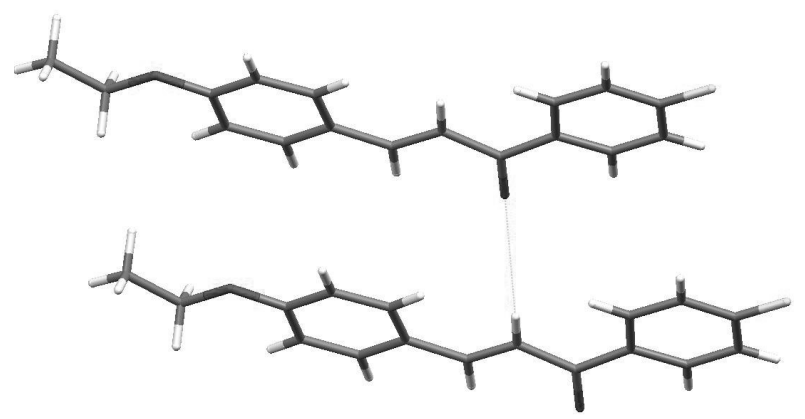

(A)

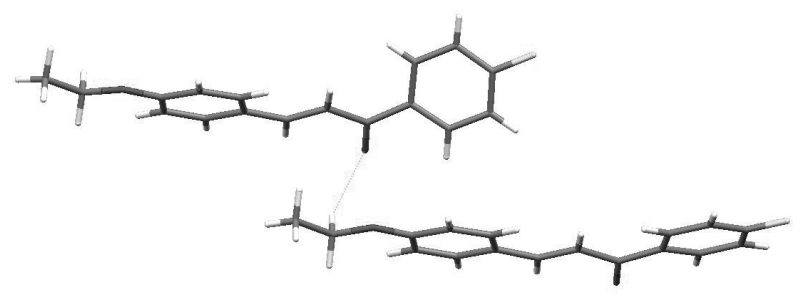

(B)

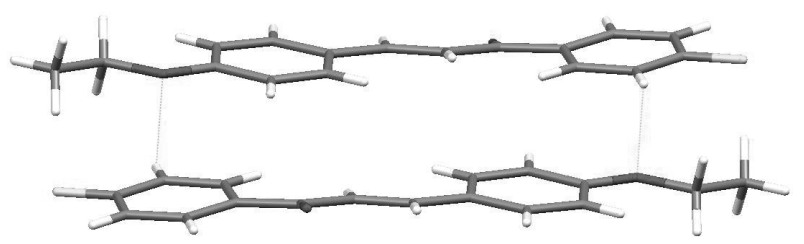

(C)

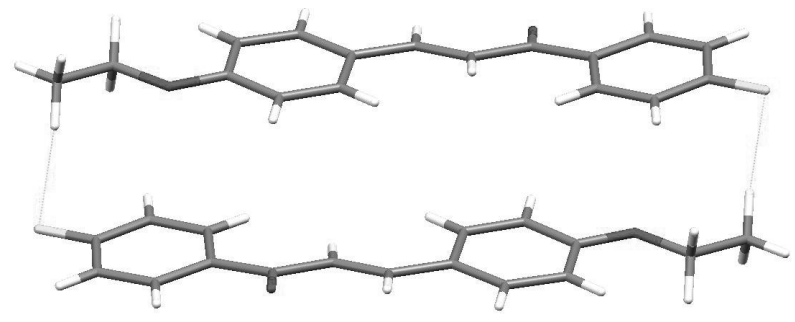

(D)

Figura 9. Ilustração das interações de hidrogênio não clássicas da chalcona $\mathrm{C}_{17} \mathrm{H}_{15} \mathrm{O}_{2} \mathrm{~F}$. (A) C8-H8...01; (B) C16-H16B...O1'; (C) $\mathrm{C} 2-$ H2O2; (D) C17-H17A...F1.

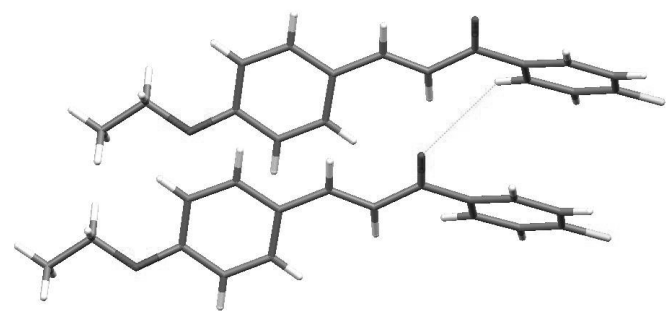

(A)

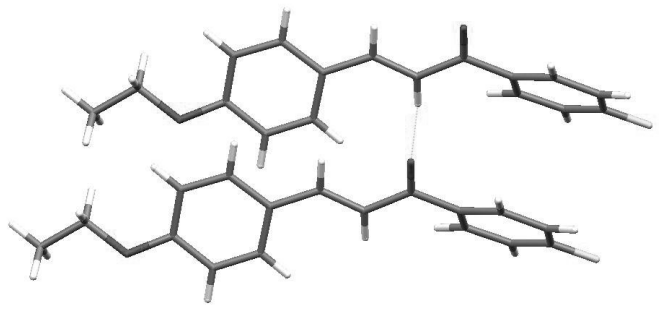

(B)

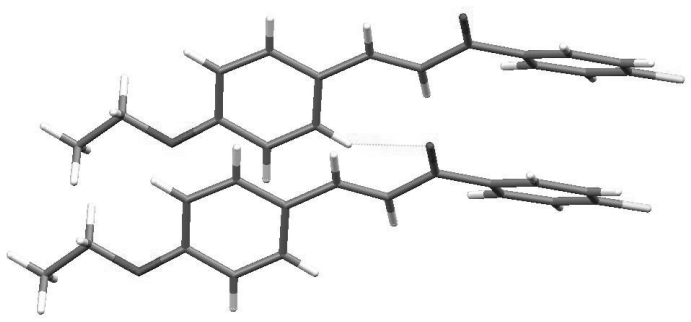

(C)

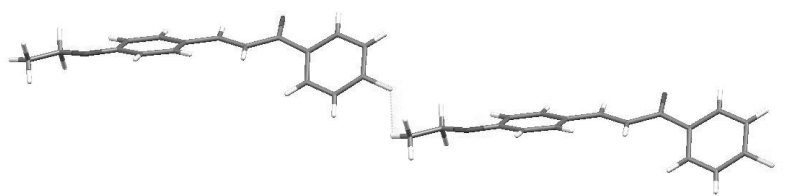

(D)

Figura 10. Ilustração das interações de hidrogênio não clássicas da chalcona $\mathrm{C}_{17} \mathrm{H}_{15} \mathrm{O}_{2} \mathrm{~F}$. (A) $\mathrm{Cl}^{\prime}-\mathrm{H} 1^{\prime} . . . \mathrm{O} 1^{\prime}$; (B) $\mathrm{C}^{\prime}$ '- $\mathrm{H}^{\prime}$ '...O1'; (C) C11'H11'...O1'; (D) C17'-H17E...F1'

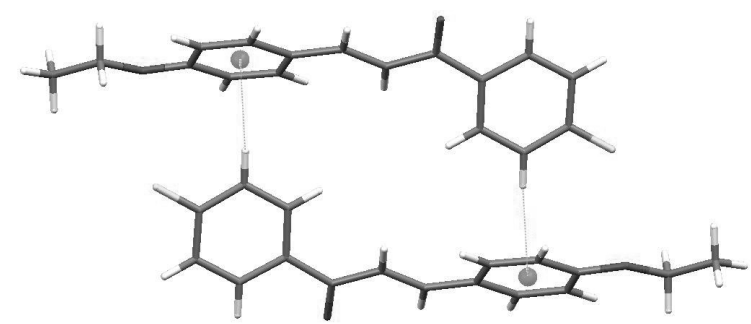

Figura 11. Ilustração da interação de hidrogênio não clássica $C 2$ '$\mathrm{H} 2$ '...Cg1. 


\section{DADOS COMPLEMENTARES DE ESPECTROSCOPIA DA CHALCONA C17H15O2F}

A pureza do composto sintetizado foi determinada através do intervalo de fusão e análise por Thin Layer Chromatography (TLC) e Gas Chromatography (GC). Para elucidação estrutural da chalcona, foi utilizada a Nuclear Magnetic Resonance for Hydrogen (1H NMR) e Carbon-13 (13C NMR) e espectroscopia de massa e infravermelho. As faixas dos pontos de fusão dos compostos foram determinadas com equipamento Microchemistry MQAPF-301, e não estão corrigidos. Os espectros de infravermelho (Figura 12) foram obtidos a partir de pastilhas de $\mathrm{KBr}$ em equipamento
FT-IR PERKINELMER modelo Spectrum Fontier. Os espectros de massa (Figura 13) foram obtidos, utilizando a cromatografia gasosa no espectrômetro de massa (GCMS) CGMS QP-210 Ultra, Shimadzu.

Os espectros de NMR, Figuras (14) e (15), de $1 \mathrm{H}$ e 13C foram obtidos no espectrômetro Bruker $500 \mathrm{MHz}$, do Instituto de Química da Universidade Federal de Goiás, usando clorofórmio deuterado como solvente comercialmente disponível a partir de Aldrich. Os desvios químicos são expressos em valores adimensionais (ppm) em relação a um padrão interno de Tetramethylsilane (TMS). A visualização dos espectros foi realizada, utilizando o programa ACD LABS 12.0.

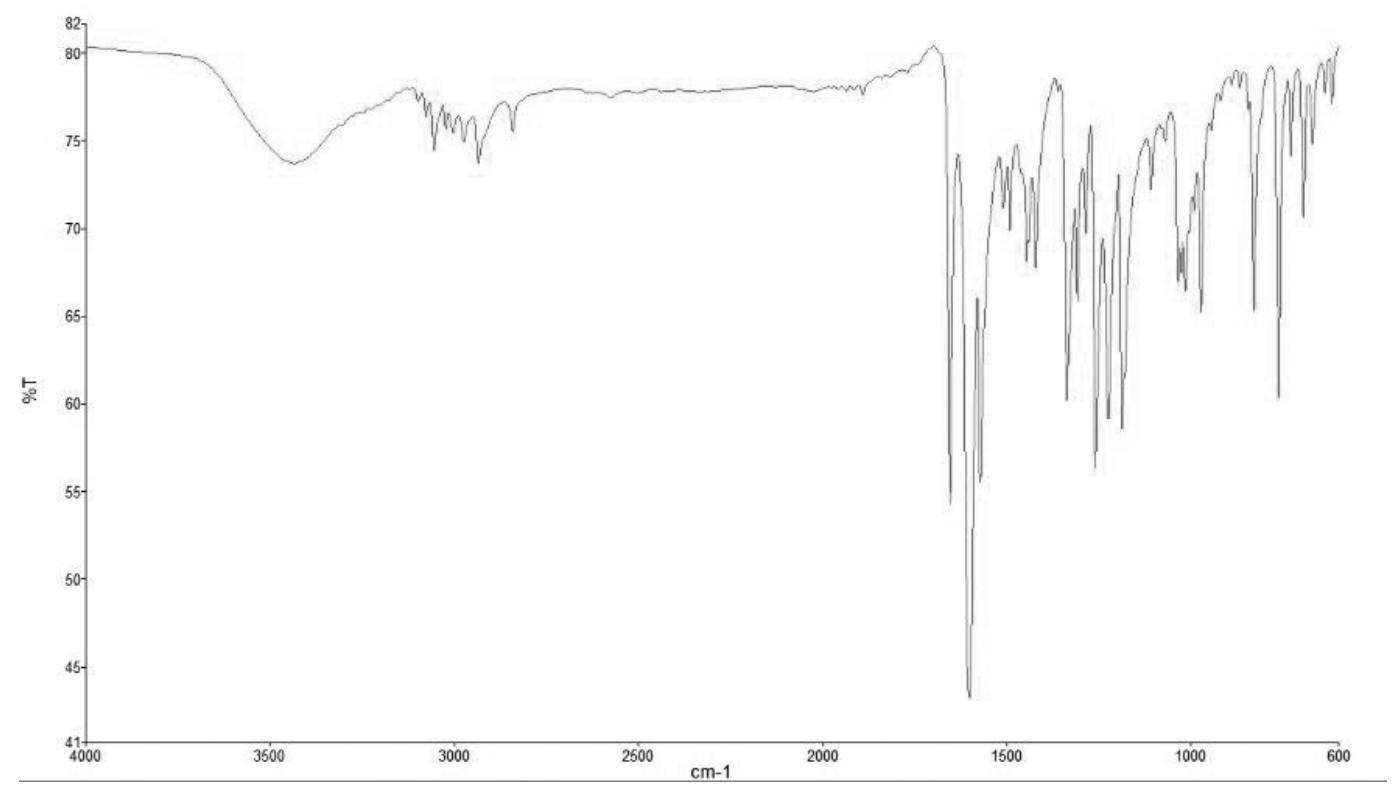

Figura 12. Espectro na região do infravermelho da chalcona $\mathrm{C}_{17} \mathrm{H}_{15} \mathrm{O}_{2} \mathrm{~F}$

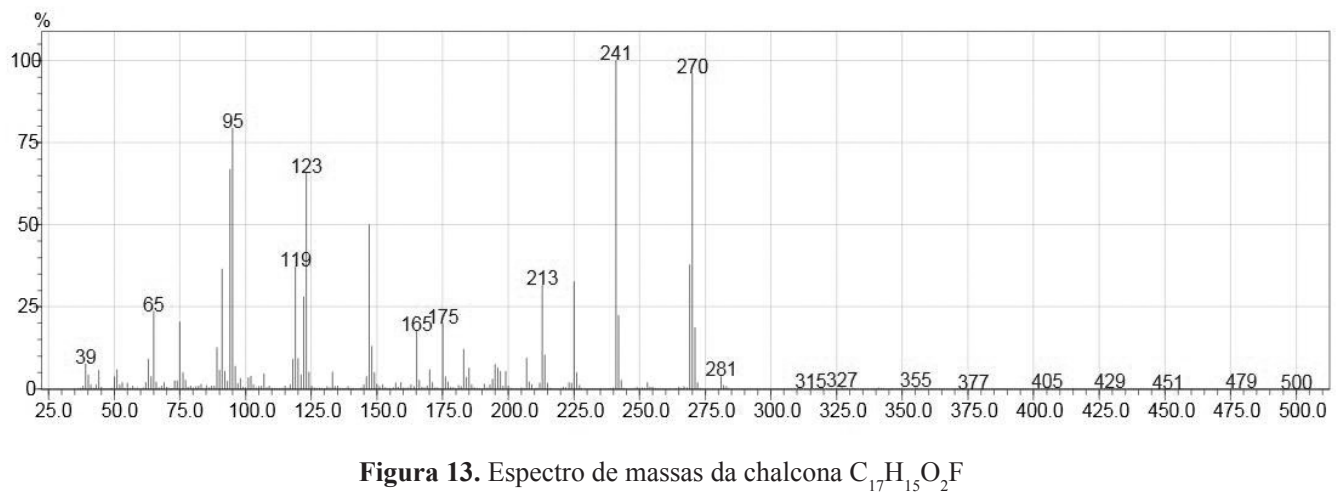


Dados Espectroscópicos: ' $\mathrm{H}$ NMR (500 MHz, $\left.\mathrm{CDCl}_{3}\right): \delta(\mathrm{ppm}) 1.47\left(\mathrm{t}, 3 \mathrm{H}, \mathrm{OCH}_{3} \mathrm{CH}_{2} \mathrm{Ph}, \mathrm{J}=7,02 \mathrm{~Hz}\right.$, $3 \mathrm{H}) 4.12$ (q, 2H, OCH $\left.\mathrm{CH}_{2} \mathrm{Ph}, \mathrm{J}=7.02 \mathrm{~Hz}\right) 6,96$ (d, $\left.2 \mathrm{H}, \mathrm{PhOCH}_{2} \mathrm{CH}_{3}, \mathrm{~J}=8.85\right)$ 7,17-7,22 (m, 2H, PhF) 7,41 (d, $1 \mathrm{H}, \mathrm{CHCO}, \mathrm{J}=15.56 \mathrm{~Hz}) 7.62\left(\mathrm{~d}, 2 \mathrm{H}, \mathrm{PhOCH}_{2} \mathrm{CH}_{3}\right.$, $\mathrm{J}=8.24$ ) 7,82 (d, 1H, CHPh, J=15.56 Hz) 8,05-8.10 (d, $2 \mathrm{H}, \mathrm{PhF}) \cdot{ }^{13} \mathrm{C}$ NMR $\left(125,7 \mathrm{MHz}, \mathrm{CDCl}_{3}\right): \delta \quad 46,02$
$(\mathrm{OCH} 3 \mathrm{CH} 2 \mathrm{Ph}) \quad 71,31\left(\mathrm{OCH}_{3} \mathrm{CH}_{2} \mathrm{Ph}\right)$ Arom. (104,84; $109,64 ; 112,42 ; 145,95 ; 151,28 ; 151,75 ; 159,81) \mathrm{C}=\mathrm{C}$. $(121,12 ; 144,64) 171.65(\mathrm{C}=\mathrm{O}) . \mathrm{IR}(\mathrm{KBr}) v 3055,2935$, $1654 ; 1601 ; 1447 ; 1308 ; 1225 ; 1157,830 ; 762 \mathrm{~cm}^{-1}$, MS (EI) $\mathrm{m} / \mathrm{z}$ (rel intensity) $270\left(\mathrm{M}^{+}, 100\right) ; 241$ (90); 225 (31); 213 (29); 175 (16); 147 (36); 123 (47); 95 (49), 75 (11); 65 (13).

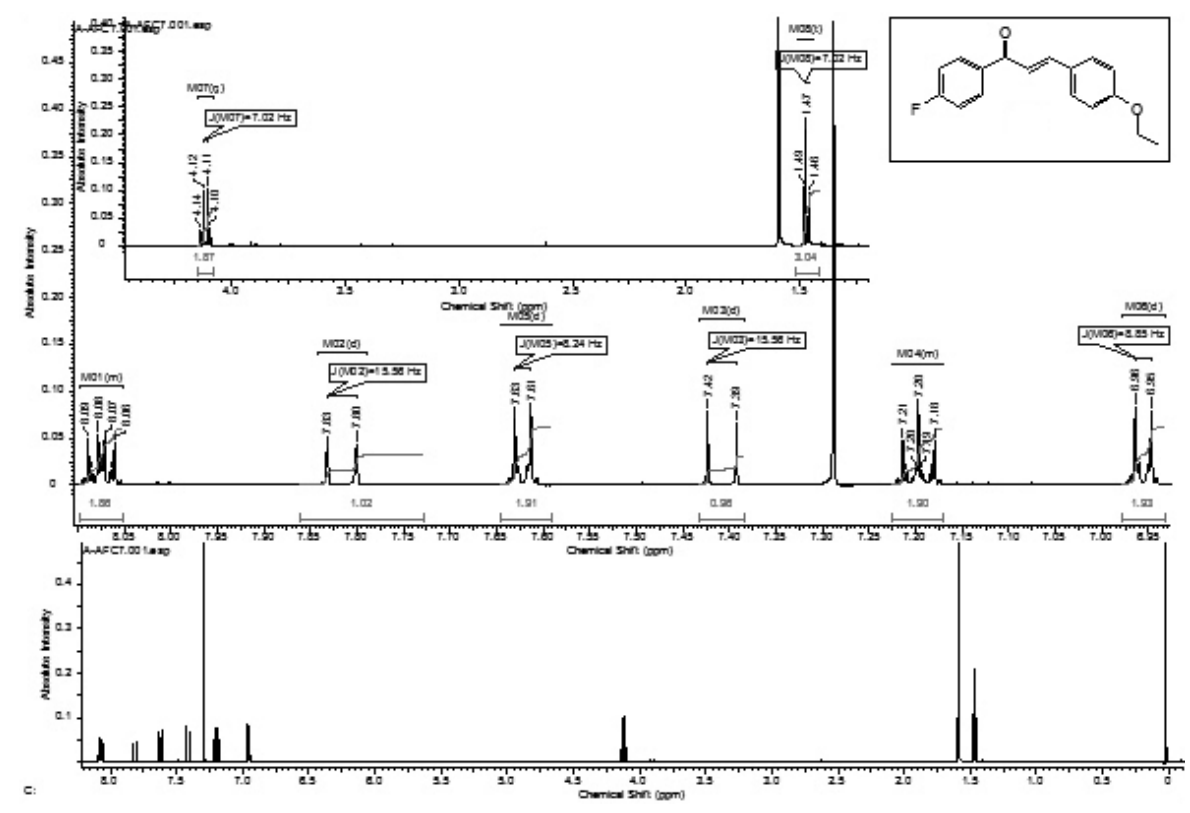

Figura 14. Espectro de ${ }^{1} \mathrm{H}-\mathrm{NMR}(500 \mathrm{MHz})$
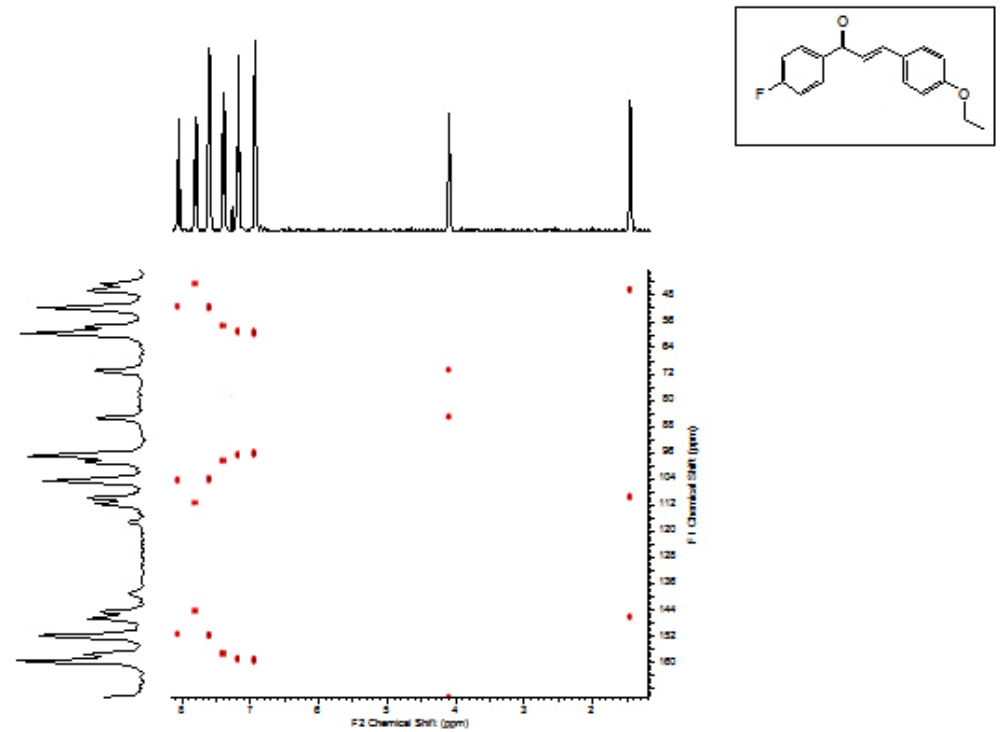

Figura 15. Mapa de contorno de Heteronuclear Single Quantum Correlation (HSQC) $\left(\mathrm{CDI}_{3}, 500 \mathrm{MHz}\right)$. 


\section{ESTUDOS DAS GEOMETRIAS MOLECULARES E POTENCIAL ENERGÉTICO}

Os parâmetros geométricos da DFT (comprimentos de ligação, ângulos de ligação e ângulos torsão) da chalcona $\mathrm{C}_{17} \mathrm{H}_{15} \mathrm{O}_{2} \mathrm{~F}$ mostram discrepâncias mais notáveis quando se observam as ligações $\mathrm{C}=\mathrm{C}$ e $\mathrm{CO}[1,34838 \AA$ e 1,35704 $\AA$ ]. O primeiro comprimento é muito maior enquanto que o segundo é levemente menor do que encontrado nas conformações da unidade assimétrica. A orientação de $\mathrm{C} 6-\mathrm{C} 7-\mathrm{C} 8=\mathrm{C} 9$ da molécula otimizada é semelhante ao encontrado na conformação $\alpha$ e define o ângulo de $177,49911^{\circ}$. Os comprimentos de ligação calculados possuem uma média absoluta a partir dos dados experimentais de $0,008 \AA$ para ambas as conformações. As diferenças podem ocorrer porque os resultados de raios-X pertençam à fase sólida, enquanto os cálculos teóricos pertençam à fase gasosa. No cristal, há existência das interações intermoleculares de moléculas de ligação. A conformação molecular da chalcona, $\mathrm{C}_{17} \mathrm{H}_{15} \mathrm{O}_{2} \mathrm{~F}$, pode ser entendida pela flexibilidade do $\mathrm{C} 6-\mathrm{C} 7-\mathrm{C} 8=\mathrm{C} 9$ e $\mathrm{C} 7-$ $\mathrm{C} 8=\mathrm{C} 9-\mathrm{C} 10$. As conformações da unidade assimétrica são diferenciadas, principalmente, pelo ângulo de torção do primeiro. A fim de revelar todas as possíveis conformações, resultantes da rotação de $\mathrm{C} 6-\mathrm{C} 7-\mathrm{C} 8=\mathrm{C} 9$, uma detalhada análise da energia potencial dimensional foi realizada para os ângulos diedros, a curva de energia potencial é mostrada na Figura 16.

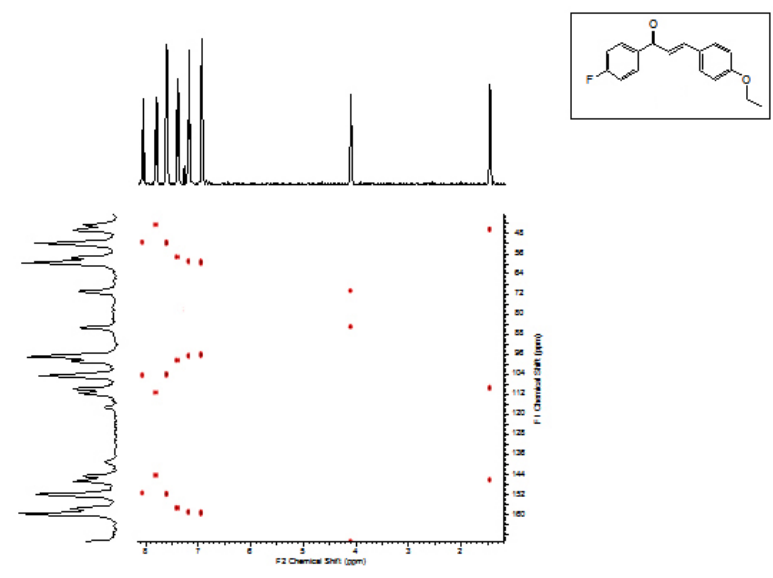

Figura 16. Varredura em torno de $\mathrm{C} 6-\mathrm{Ph} 1$.
Nesta curva, pode-se observar que as conformações são confinadas na área de potencial estável bem como a barreira de potencial de $0,147532 \mathrm{Kcal} / \mathrm{mol}$. Por isso, a conformação molecular possível se restringe dentro destes valores, tais como nas moléculas $\alpha$ e $\beta$. A configuração planar da chalcona $\alpha, \mathrm{C}_{17} \mathrm{H}_{15} \mathrm{O}_{2} \mathrm{~F}$, é a curva global mínima $\left[\mathrm{C} 6-\mathrm{C} 7-\mathrm{C} 8=\mathrm{C} 9=177,176^{\circ}\right]$ e a conformação $\beta$ é a mais próxima deste estado. A energia da conformação $\alpha$ é mais baixa que a da conformação $\beta$ em cerca $0,38057315 \mathrm{Kcal}$ / mol, o que indica que ambas são estáveis e igualmente possíveis no estado solúvel. O aumento da curva de energia está relacionado com a repulsão entre os átomos de hidrogênio. A curva mostra ainda uma conformação meta-estável, quando o ângulo de torção é $47,176^{\circ}$, com a distância entre $\mathrm{H} 5$ e $\mathrm{H} 8$ de 2,67720 A. Energicamente, a rotação completa de $\mathrm{C} 6-\mathrm{C} 7-\mathrm{C} 8=\mathrm{C} 9$ é um processo envolvendo elevada energia de moléculas com uma barreira energética de $52,0 \mathrm{kcal} / \mathrm{mol}$. A direcionalidade da interação $\mathrm{CH} . . . \pi$ envolvendo a conformação $\beta$ da chalcona $\mathrm{C}_{17} \mathrm{H}_{15} \mathrm{O}_{2} \mathrm{~F}$ é uma regra para a compreensão da estabilidade do sistema na estrutura cristalina. A varredura da rotação de $\mathrm{C} 6-\mathrm{C} 7-\mathrm{C} 8=\mathrm{C} 9$, utilizando a ligação não clássica, é mostrada na Figura 17.

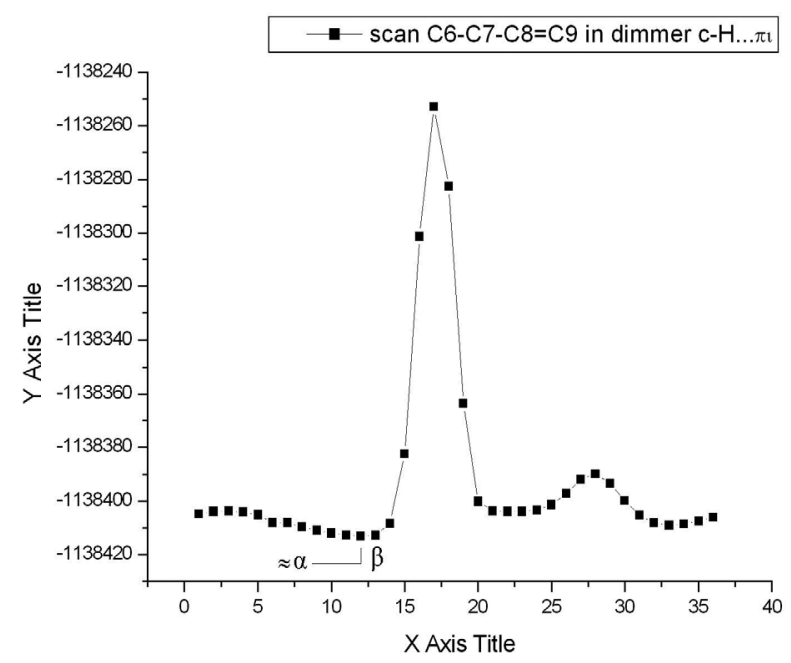

Figura 17. Varredura em torno $\mathrm{C} 6-\mathrm{Ph} 1$ na ligação $\mathrm{C}-\mathrm{H}$... $\pi$ 
Tal como esperado, as duas conformações $(\alpha$ e $\beta)$ são energeticamente próximas, mas a conformação $\beta$ é a curva global mínima. A ligação $\mathrm{CH} \cdots \pi$ é direcional e a conformação $\beta$ é a conformação mais estável, que liga o C2'-H2' com o centroide da molécula adjacente a $126,15^{\circ}$. A curva mostra dois estado de alta energia, a instabilidade do primeiro está relacionada com a grande repulsão entre H2' e H11', enquanto o segundo é relacionado com a intrarrepulsão entre H2 e H9. Os arranjos meta-estáveis são observados quando o ângulo é $-29,07639^{\circ}$. Este estado reduz a repulsão de Van Der Walls mas não otimiza os contatos intermoleculares, tais como a conformação $\beta$. As propriedades termodinâmicas são indicativos de estabilidade da ligação $\mathrm{CH}$... $\pi$. As conformações $\alpha$ e $\beta$ existem porque não houve frequência negativa.

O MEP está associado com a densidade eletrônica, sendo uma propriedade importante para explicar sítios de reatividade, bem como suas interações. O MEP negativo corresponde às zonas de elevada densidade eletrônica, que é visto na proximidade do flúor, átomos de oxigênio, carbonila e etoxila.

\section{Considerações Finais}

A estrutura cristalográfica da chalcona $\mathrm{C}_{17} \mathrm{H}_{15} \mathrm{O}_{2} \mathrm{~F}$ demonstra a ausência de ligação de hidrogênio clássica, tanto intramolecular quanto intermolecular, ou seja, ambas conformações observadas apresentam apenas ligações de hidrogênio fracas, intermoleculares, que são consistentes com a baixa barreira de potencial calculada de $0,147532 \mathrm{Kcal} / \mathrm{mol}$.

A conformação $\beta$ apresenta uma interação fraca a mais que a $\alpha$, mas possui três interações, envolvendo o oxigênio O1' como aceitador, diminuindo, assim, o valor energético delas. Cabe destacar que nesta conformação, a interação C2'-H2'...Cg1, que forma um dímero, é determinante na formação de uma cadeia infinita destes dímeros.

Outro aspecto relevante associado à interação C2'H2' ...Cg1 na conformação $\beta$, que levou a torção C6-C7$\mathrm{C} 8=\mathrm{C} 9$, é a diferença conformacional indicada na Figura 6. O valor de $6,25^{\circ}$ observado para a diferença do ângulo de torção, é consistente com esta interação $\mathrm{C}-\mathrm{H}$...Cg observada. Finalmente, a multiplicidade conformacional da unidade assimétrica é coerente com o baixo valor energético das interações fracas de cada conformação.
Os dados espectroscópicos, são consistentes com a estrutura cristalográfica elucidada. Da mesma forma, os resultados obtidos por DFT indicam a estabilidade para ambas conformações, Figura 16, observadas na unidade assimétrica. Estes dados indicam ainda, que a varredura $\mathrm{C}-\mathrm{H} . . . \pi(\mathrm{C} 6-\mathrm{C} 7-\mathrm{C} 8=\mathrm{C} 9)$, tem intervalos de valores teóricos coerentes com aqueles experimentais observados. Dessa forma, os cálculos de DFT não apenas explicam a observação de duas conformações para a chalcona $\mathrm{C}_{17} \mathrm{H}_{15} \mathrm{O}_{2} \mathrm{~F}$, como indicam a consistência dos parâmetros estruturais (distâncias, ângulos de ligação e ângulos dihedrais) neles observados.

Este estudo elucida a estrutura cristalina de uma nova chalcona Fluorada com Z'>1, (conformação $\alpha$ e $\beta$ ), e demonstra que o empacotamento do cristal e a análise das energias são explicações simples para compreender suas estruturas moleculares. Por conseguinte, conclui-se que a baixa simetria; a interação dimérica não clássica; a pequena diferença de energia entre as conformações dominam a estabilidade do sistema. Na estrutura do cristal, $\mathrm{C}_{17} \mathrm{H}_{15} \mathrm{O}_{2} \mathrm{~F}, \alpha$ e $\beta$ tem conjunto próprio supramolecular; a primeira é planar, dimeriza e está disposta em cadeias infinitas, enquanto a ligação $\mathrm{CH} . . . \pi$ é importante para estabilizar conformação $\beta$ dimerizada. Os resultados calculados revelam que a diferença de energia entre duas conformações é de cerca de $0,345 \mathrm{kcal} / \mathrm{mol}$, indicando que ambas são igualmente possíveis em estado solúvel. O gráfico da varredura mostra que a energia potencial das duas conformações está próxima dos mínimos.

Considerando a identidade estrutural da chalcona $\mathrm{C}_{17} \mathrm{H}_{15} \mathrm{O}_{2} \mathrm{~F}$ com outras chalconas que apresentam atividade anticarcinogênica, seria de grande valor científico que testes biológicos fossem realizados; claro que os testes anticarcinogênicos não excluem os testes clássicos para outras patologias.

\section{Referências Bibliográficas}

1. Anderson, K. M.; Goeta, A. E.; Steed, J. W. Supramolecular Synthon Frustation Leads to Crystal Strctures With Z'>1. Crystal Growth \& Design. vol. 8. p. 2517-2524. 2008.

2. Bandgar, B. P.; Gawande, R. G.; Bodade, C. N. Synthesis and biological evaluation of simple methoxylated chalcones as anticancer, anti inflammatory and antioxidant agents.Bioorganic \& Medical Chemistry. vol. 18. p. 1364-1370. Fev. de 2010. 
3. Camargo, L. T. F. M.; Camargo, A. J.; Napolitano, H. B. Estudo químico quântico da atividade da indolo $(2,1 \mathrm{~b})$ quinazolina e seus derivados análogos contra o câncer de mama. Processos Químicos. p. 61. 2008.

4. Cullity, B. D. Elements of x- ray Diffraction. 3.ed. AddisonWesley Publishing Company Inc, 2012.

5. Dhar, D. N. The Chemistry of Chalcones and Related Compounds. New York: Wiley, 1981.

6. Dimmock, J. R.; Elias, D. W.; Beazely, M. A.; Kandepu, N. M. Bioactivities of chalcones.Curr. Med. Chem. vol. 6. p. 1125-1149. 1999.

7. Farrugia, L. J. Wingx and ORTEP for Windows: an update. J. Appl. Crystallogr. vol. 45.p. 849-854. 2012.

8. Frisch, M. J.; Trucks, G. W.; Schlegel, H. B.; Scuseria, G. E.; Robb, M. A.; Cheeseman, J. R.; Montgomery, Jr., J. A.; Vreven, T.; Kudin, K. N.; Burant, J. C.; Millam, J. M.; Iyengar, S. S.; Tomasi, J.; Barone, V.; Mennucci, B.; Cossi, M.; Scalmani, G.; R. Gaussian 03 Revision C 02.Inc. Wallingford CT, 2004.

9. Giacovazzo, C.; Mônaco, H. L.; Artioli, G.; Viterbo, D.; Milanesio, M.; Gilli, G.; Gilli, P.; Zanotti, G.; Ferraris, G.. Fundamentals of Crystallography. 3. ed .New York:IUCROxford University Press, 2011.

10. Glusker, J. P.; Trueblood, K. N. Crystal Structure Analysis a Primer. 3. ed. Oxford University Press, 2010.

11. Jensen, W. P.; Palenik, G. J.; Suh, I. H. The history of molecular structure determination viewed through the nobel prizes. J. CHEM. vol. 80. p. 753. 2003.

12. Kamal, A.; Mallareddy, A.; Suresh, P.; Shaik, T.B.; Nayak, V.L.; Kishor, C.; Shetti, R.V.C.R.N.C.; Rao, N.S.; Tamboli, J.R.; Krishna, S.R.; Addlagatta, A. Synthesis of chalconeamidobeenzothiazole conjugates as antimitotic and apoptotic inducing agents. Bioorganic \& Medicinal Chemistry. vol. 20.1 de june de 2012. p. 3480-3492.

13. Liu, X. F.; Zheng, C. J.; Sun, L. P.; Liu, X. K.; Piao, H. R. Synthesis of new chalcone derivatives bearing 2,4-thiazolidinedione and benzoic acid moieties as potencial antibacterial agents. Eur.J.Med.Chem. vol. 46. p. 3469-3473. 2011.

14. Nakamura, C.; Kawasaki, N.; Miyataka, H.; Jayachandran, E.; Kim, I.H.; Kirk, K.L.; Taguchi, T.; Takeuchi, Y.; Hori, H.; Satoh,
T. Synthesis and Biological Activities of Fluorinated Chalcone Derivatives. Bioorganic \& Medicinal Chemistry. vol. 10. p. 699706. 2002.

15. Nowakowska, Z. A review of anti-infective and anti-inflammatory chalcones. Eur. J. Med. Chem. vol. 42. p. 125-127. 2007.

16. Schwarzer, A.; Weber, E. (E)-1,3-Bis(2,3,4,5,6pentafluorophenyl)-prop-2-en-1-one. Acta Cryst. vol. E66. p. 1931. 2010.

17. Sheldrick, G. M. Shelxs-97: Program for the solution of crystal structures. Germany: University of Gottingen, 1990.

18. ShmuelI, U. Theories and Techniques of Crystal Structure Determination. New York: Oxford University Press- IUCr, 2007.

19. Steed, J. W. Should solid-state molecular packing have to obey the rules of crystallographic symmetry? CrystEngComm. vol. 5. p. 169-179. 2003.

20. Wu, J.; Li, J.; Cai, Y.; Pan, Y.; Ye, F.; Zhang, Y.; Zhao, Y.; Yang, S.; Li, X.; Liang, G.Evaluation and Discovery of Novel Synthetic Chalcone Derivatives as Anti-Inflammatory Agents. J.Med.Chem. vol. 54. p. 8110-8123. 2011.

\section{Cássio C. Almeida ${ }^{1,2, *}$, Ademir J. Camargo', 'Gilberto B. Aquino' \& Hamilton B. Napolitano'}

${ }^{1}$ Grupo de Química Teórica e Estrutural de Anápolis, Ciências Exatas e Tecnológicas. Universidade Estadual de Goiás, CP 459, 75001-970 Anápolis, GO, Brasil.

${ }^{2}$ Instituto Federal Goiano - Campus Urutaí.

E-mail: cassiocirilo@gmail.com 
